J. Lake Sci. (湖泊科学) , 2018, 30(4): 1004-1011

DOI 10. 18307/2018. 0413

(C) 2018 by Journal of Lake Sciences

\title{
典型湖泊天然有机质与四环素的相互作用
}

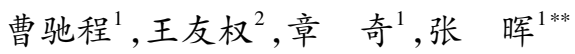 \\ ( 1 : 东南大学公共卫生学院环境科学工程教育部重点实验室,南京 210009) \\ (2: 南京林业大学生物与环境学院, 江苏省环境工程重点实验室,南京 210037)
}

\begin{abstract}
摘 要: 采用苂光滴定法研究四环素 (tetracycline, TC) 与太湖溶解性有机质 (dissolved organic matter, DOM) 和玄武湖 DOM 的相互作用. 三维菼光光谱结合平行因子分析显示, 2 个湖泊的 DOM 含有 3 个菼光组分: 类富里酸组分 C1、类色氨 酸组分 $\mathrm{C} 2$ 和类络氨酸组分 $\mathrm{C} 3$. 其中 $\mathrm{C} 2$ 的荧光强度远高于 $\mathrm{C} 1$ 和 $\mathrm{C} 3$, 是 DOM 的主要荧光组成. 3 个苂光组分与 $\mathrm{TC}$ 发生 了不同程度的静态猝灭, 特别当 TC 浓度为 $45.5 \mu \mathrm{mol} / \mathrm{L}$ 时, 类蛋白组分的苂光强度完全被猝灭 (100\%), 并且猝灭作用改 变了 DOM 分子的微环境极性. 同步菼光光谱联合二维相关图谱进一步表明类色氨酸组分优先和 TC 发生猝灭作用, 其次 为类络氨酸组分和类富里酸组分. Ryan-Weber 方程适于拟合 DOM 与 TC 的猝灭过程, 2 个湖泊的 DOM 中 3 个苂光组分的 络合常数 $\lg K$ 值范围为 $5.05 \sim 5.85$, 大小顺序为 $\mathrm{C} 2>\mathrm{C} 3>\mathrm{C} 1$. 因此, 类蛋白组分为主的 DOM 对 TC 的络合作用大于类腐殖 组分为主的 DOM, 影响抗生素在湖泊水体中的生物有效性和生态毒性.
\end{abstract}

关键词：四环素;溶解性有机质;荧光猝灭;平行因子分析;二维相关图谱; 太湖; 玄武湖

\section{Interactions of tetracycline and dissolved organic matter from freshwater lakes}

\author{
CAO Chicheng ${ }^{1}$, WANG Youquan ${ }^{2}$, ZHANG Qi ${ }^{1} \&$ ZHANG Hui ${ }^{1 * *}$ \\ (1: Key Laboratory of Environmental Medicine Engineering, Ministry of Education, School of Public Health, Southeast Uni- \\ versity, Nanjing 210009 , P.R.China) \\ (2: Key Laboratory of Environmental Engineering of Jiangsu Province, College of Biology and the Environment, Nanjing For- \\ estry University, Nanjing 210037, P.R.China)
}

Abstract: Interactions of tetracycline (TC) with dissolved organic matter (DOM) collected from Lake Taihu and Lake Xuanwu were investigated through fluorescence quenching. Three fluorescent DOM components, fulvic-( C1), tryptophan-( C2), and tyrosine-like ( C3 ) component, were identified in DOM via excitation emission matrix spectra with parallel factor analysis (EEMPARAFAC). Results from fluorescence quenching titration showed that the fluorescence of 3 components was quenched at different degree by TC through static quenching. When the TC concentration increased to $45.5 \mu \mathrm{mol} / \mathrm{L}$, the whole florescence of C2 and C3 were almost quenched, while $20 \%$ of $\mathrm{C} 1$ fluorescence was inaccessible. The quenching also induced conformational changes in DOM molecules. Synchronous fluorescence spectra combined with two dimensional correlation spectroscopy (2D-COS) further revealed that the complexation firstly occurred for tryptophan-like substance, followed by tyrosine- and fulvic-like substance. The associated complexing constants $(\lg K)$ of 3 components with TC were in the range of 5.05-5.85, followed the order of $\mathrm{C} 2>\mathrm{C} 3>$ C1. The strong binding ability of protein-like substances confirmed their prominent role in the TC-DOM complexation. These results highlight the significant impact of DOM on the bioavailability and ecotoxicity of antibiotics in freshwater lakes.

Keywords: Tetracycline; dissolved organic matter; fluorescence quenching; 2D-COS; PARAFAC; Lake Taihu; Lake Xuanwu

溶解性有机质 (dissolved organic matter, DOM) 是细菌、藻类和植物经过复杂生化过程形成的不均匀有 机混合物, 在水陆生态系统中具有重要作用 ${ }^{[1-2]}$. DOM 中含有多种亲水、疏水官能团, 如羧基、羟基、酚基和 羰基等,可通过化学鳌合、疏水分配、极性作用以及氢键等机制与有机污染物相互作用,从而影响污染物在

* 东南大学通识课程项目 (1125000131) 和中央高校基本科研业务费资助项目 (2242017K40041) 联合资助. 201711-13 收稿; 2018-01-02 收修改稿. 曹驰程 (1992 ), 女, 硕士研究生;E-mail : caochicheng2010@163.com.

** 通信作者;E-mail:13851553232@163.com. 
环境中的形态、迁移、生物有效性和风险 ${ }^{[3-4]}$.

四环素类药物 (tetracycline antibiotics, TCs) 是一种典型的抗生素, 广泛应用于人体和动物的医疗及畜禽 养殖. TCs 在生物体内代谢不完全,约 30\% 90\% 通过废水和粪便进人水环境中, 导致 TCs 在地表水中残留 $(0.13 \sim 0.51 \mu \mathrm{g} / \mathrm{L})$, 诱导耐药细菌的产生和抗性基因的形成, 对人体健康和生态环境造成潜在风险 ${ }^{[5-6]}$. 近 年来, 菼光猝灭因其高敏感性、简便性和无损性等优点被广泛用来研究 DOM 与污染物的相互作用 ${ }^{[7-8]}$. 其原 理为苂光团与猝灭剂接触时, 可通过激发态碰撞、能量转移和稳定态形成等机制导致荧光团苂光性降低. DOM 成分复杂, 不同苂光团的光谱往往会重叠, 但采用三维荧光图谱结合平行因子模型 (excitation emission matrix fluorescence with parallel factor analysis, EEM-PARAFAC) 可以有效地分析独立荧光组分与污染物之间 的作用特征 ${ }^{[9-10]}$. 此外, 结合二维相关光谱分析 (two-dimensional correlation spectroscopy, 2D-COS) 可进一步 定性表征不同 DOM 组分与污染物之间的作用顺序 ${ }^{[11-12]}$. 因此, 联用多种光谱手段可从不同层面阐述 DOM 与四环素之间的相互作用特征 ${ }^{[13-14]}$.

本研究选择玄武湖和太湖的 DOM 作为研究对象, 选择湖泊水体污染物备受关注的抗生素 $\mathrm{TCs}$ (文献报 道太湖水体 $\mathrm{TC}$ 浓度为 $0 \sim 87.9 \mathrm{ng} / \mathrm{L}$, 玄武湖水体 TC 浓度为 $0 \sim 5.21 \mathrm{ng} / \mathrm{L})^{[15-16]}$, 采用苂光猝灭滴定, 结合同 步荧光光谱、2D-COS、EEM-PARAFAC 以及紫光可见光谱 (UV-Vis), 研究 TCs 与湖泊 DOM 之间的相互作用 特点, 揭示天然有机质 DOM 与 TCs 相互作用的微观机制, 以期为理解 TCs 在湖泊生态系统中的迁移转化和 生态风险提供理论依据.

\section{1 材料和方法}

\section{1 药剂准备和 DOM 采集}

盐酸四环素 (Tetracycline hydrochloride, TC) 纯度为 $>99.9 \%$, 购自美国 Sigma Aldrich 公司, 其他试剂均为 分析纯,用水为 Milli-Q 超纯水.

2017 年 6 月分别采集太湖 $\left(30^{\circ} 56^{\prime} \sim 31^{\circ} 34^{\prime} \mathrm{N}, 119^{\circ} 54^{\prime} \sim 120^{\circ} 36^{\prime} \mathrm{E}\right.$ ) 和玄武湖 $\left(32^{\circ} 34^{\prime} \sim 32^{\circ} 50^{\prime} \mathrm{N}, 118^{\circ} 47^{\prime} \sim\right.$ $\left.118^{\circ} 49^{\prime} \mathrm{E}\right)$ 的表层水, 用 $\mathrm{GF} / \mathrm{F}$ 玻璃纤维滤膜 $\left(450^{\circ} \mathrm{C}\right.$ 灼烧 $\left.4 \mathrm{~h}\right)$ 过滤, 得到太湖 DOM 和玄武湖 DOM. 将 2 个湖 泊的 DOM 在 $4^{\circ} \mathrm{C}$ 条件下透析 $24 \mathrm{~h}(3500 \mathrm{Da})$, 去除无机盐和有机小分子. 最后, 用 Milli-Q 超纯水稀释使得 2 个湖泊 DOM 的溶解性有机碳 (dissolved organic carbon, DOC) 浓度为 $30 \mathrm{mg} / \mathrm{L}$, 冷冻保存.

\section{2 荧光猝灭滴定}

分别取 $15 \mathrm{ml} 2$ 个湖泊的 DOM 到 $50 \mathrm{ml}$ 棕色玻璃管中, 再向管中添加一定量的 TC 储备液和 $0.02 \mathrm{~mol} / \mathrm{L}$ 的磷酸盐缓冲液 $(\mathrm{pH}=7.0$ ), 使最终反应体系为: 总体积 $30 \mathrm{ml}$, DOC 浓度为 $15 \mathrm{mg} / \mathrm{L}$, TC 浓度为 $0 \sim 70 \mathrm{mg} / \mathrm{L}$. 用聚四氟乙烯塞密封后, $25^{\circ} \mathrm{C}$ 下恒温震荡. 预实验表明 DOM 与 TC 在 $8 \mathrm{~h}$ 内达到反应平衡. 对照实验表明反 应过程中挥发和吸附损失小于 $1 \%$. 平衡后测定 DOM-TC 混合液的紫外和荧光光谱.

\section{3 分析方法}

DOC 值由 TOC-V $\mathrm{cph}$ 型总有机碳分析仪 (岛津, 日本) 测定; UV-Vis 光谱由 UV-2550 型紫外分光光度计 (岛津, 日本) 测定, 以 Milli- Q 纯水为空白, 扫描波段为 $200 \sim 800 \mathrm{~nm}$, 间隔为 $1 \mathrm{~nm}$, 以 $700 \mathrm{~nm}$ 处吸收值执行 基线校正. 同步荧光光谱和 EEM 由 F-7000 型荧光分光光度计 (日立, 日本) 测定, 激发光源为 $700 \mathrm{~V}$ 的光电 倍增管和 $150 \mathrm{~W}$ 氙弧灯. 同步苂光光谱的扫描激发波长为 $200 \sim 450 \mathrm{~nm}$, 间隔 $1 \mathrm{~nm}$, 发射波长与激发波长差 值 $\Delta \lambda=60 \mathrm{~nm}$, 扫描速度 $240 \mathrm{~nm} / \mathrm{min}$. EEM 的扫描激发波长 $200 \sim 450 \mathrm{~nm}$, 间隔 $5 \mathrm{~nm}$, 扫描发射波长 $250 \sim 550$ $\mathrm{nm}$, 间隔 $1 \mathrm{~nm}$, 激发和发射夹缝宽度 $5 \mathrm{~nm}$, 扫描速度 $1200 \mathrm{~nm} / \mathrm{min}$. 采集 EEM 后, 按仪器方法消除误差, 并通 过瑞利效应赋值和拉曼散射综合区域标准化消除干扰峰. 采用 Matlab(2012a) 软件中的 drEEM 工具箱消除 内滤效应,并将荧光强度标准化为激发波长 $350 \mathrm{~nm}$ 处的拉曼信号强度 $\left(\mathrm{RU}_{350}\right)^{[17]}$.

\section{4 模型分析}

1.4.1 二维相关图谱 (2D-COS) 分析 二维相关图谱相较于一维图谱, 不仅可分辨出重叠峰和隐藏峰, 并且可 通过信号峰之间变化的关系来研究苂光团和猝灭剂的相互作用 ${ }^{[18]}$. 对 TC 浓度不同的 DOM 同步苂光光谱 进行 2D-COS 分析, 用 2D Shige version 1.3 (关西大学, 日本) 软件进行标准化分析, 并将结果用 Matlab (2012a) 软件绘制图谱. 
1.4.2 PARAFAC 分析 PARAFAC 分析将获得的苂光强度以三向数据矩阵 $I \times J \times K($ 样品数量 $\times$ 激发波长 $\times$ 发 射波长) 形式输出, 并通过交替最小二乘算法把所有样品的整个荧光数据矩阵分解成 3 个线性项和 1 个残 留数组 ${ }^{[2]}$. PARAFAC 的矩阵公式如下:

$$
X_{j i k}=\sum_{f=1}^{F} a_{i f} \cdot b_{i f} \cdot c_{k f}+e_{j i k}
$$

式中, $i=1 、 2 、 \cdots 、 I ; j=1 、 2 、 \cdots 、 J ; k=1 、 2 、 \cdots 、 K ; f$ 为某个苂光组分; $F$ 为苂光组分总数目; $e$ 为残差. 用 drEEM 工具箱对样品的 EEM 进行运算, 并对运算结果用残差分布和 “S4T6C3” 算法验证. 设定每个荧光组 分的最大苂光强度 $\left(F_{\text {max }}\right)$ 与其相应浓度成固定比例.

1.4.3 Ryan-Weber 模型拟合 PARAFAC 分离的 DOM 组分与 TC 之间的反应模型使用 Ryan-Weber 非线性等 式拟合相关参数 ${ }^{[19]}$. 该模型基于静态猝灭过程. 设定每个苂光组分初始苂光强度为 100 , Ryan-Weber 非线性 模型如式(2)所示:

$$
F=\frac{\left(F_{\text {end }}-100\right)}{2 K \cdot C_{\mathrm{L}}}\left(K \cdot C_{\mathrm{L}}+K \cdot C_{\mathrm{TC}}+1-\sqrt{\left(K \cdot C_{\mathrm{L}}+K \cdot C_{\mathrm{TC}}+1\right)^{2}-4 K \cdot C_{\mathrm{L}} \cdot C_{\mathrm{TC}}}\right)+100
$$

式中, $C_{\mathrm{TC}}$ 为 $\mathrm{TC}$ 浓度 $(\mu \mathrm{mol} / \mathrm{L}), F$ 为苂光组分与对应浓度 $\mathrm{TC}$ 结合后的苂光强度相对初始苂光强度的百分 值, $F_{\text {end }}$ 为滴定达到理论终点时的苂光组分的苂光强度相对初始苂光强度的百分值, $C_{\mathrm{L}}$ 为苂光组分中配体 $\mathrm{L}$ 的浓度 $(\mu \mathrm{mol} / \mathrm{L}), K$ 为络合常数. 使用 1 stopt 1.5 软件计算得出 $K 、 C_{\mathrm{L}}$ 和 $F_{\text {end }}$.

\section{2 结果与讨论}

\subsection{2 个湖泊 DOM 的三维荧光光谱特征}

一般最大发射波长小于 $380 \mathrm{~nm}$ 的苂光峰被认为是含有羟基和氨基等供电子基团的苯环结构,而大于 380 $\mathrm{nm}$ 的峰则与多环芳香结构有关 ${ }^{[20]}$. 参考前人研究 ${ }^{[11]}$, 太湖 DOM 的 EEM 图中 A 峰 (Ex/Em $\left.275 \mathrm{~nm} / 325 \mathrm{~nm}\right)$ 和 B 峰 $(\mathrm{Ex} / \mathrm{Em} 230 \mathrm{~nm} / 330 \mathrm{~nm})$ 可归为类蛋白物质, 而位于 $\mathrm{Ex} / \mathrm{Em} 330 \mathrm{~nm} / 425 \mathrm{~nm}$ 的 C 峰主要是类腐殖物质 (图 1a). 玄武湖 DOM 同样具有 3 个峰, 其中 A 峰和 B 峰的位置与太湖 DOM 相似, 而 D 峰位于 Ex/Em 375 $\mathrm{nm} / 500 \mathrm{~nm}$, 可表征为结构更紧密的类腐殖物质 (图 1b). 2 个湖泊 DOM 中 3 个苂光峰的强度大小为 A 峰 $>$ B 峰 $>C(D)$ 峰, 表明类蛋白物质是 2 个湖泊 DOM 的主要组成成分, 而类腐殖物质较少. 太湖和玄武湖 DOM 与 有机质结构和成熟度有关的苂光峰比值 $r(\mathrm{~A}, \mathrm{C})$ 值分别为 1.25 和 $1.35^{[21]}$. 据文献报道 ${ }^{[22]}$, 在洁净水体中类 蛋白苂光峰强度很低, 而随着受污染程度增加, 类蛋白苂光峰强度逐渐升高. 因此, 2 个湖泊存在一定程度的 水质污染, 与前人调查结果一致 ${ }^{[21]}$. 本研究采样时间为夏季, 2 个湖泊中浮游藻类和水生植物因富营养化而 过量生长, 其自身代谢和残体降解会释放大量类蛋白 DOM 进人水体中, 致使类蛋白苂光组分浓度升高 ${ }^{[2,4]}$.
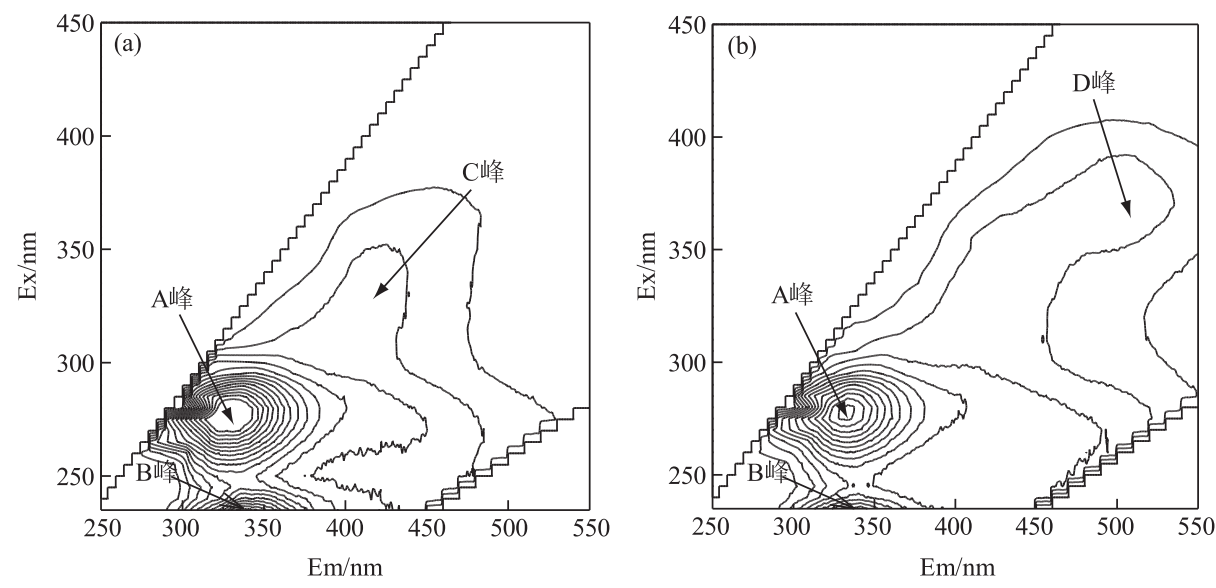

图 1 太湖 $\operatorname{DOM}(\mathrm{a})$ 和玄武湖 $\operatorname{DOM}(\mathrm{b})$ 的三维荧光图谱

Fig.1 Excitation-emission matrix spectra of DOM in Lake Taihu(a) and Lake Xuanwu(b) 


\subsection{2 个湖泊 DOM 与 TC 的荧光猝灭效应}

随着 TC 浓度增加, 太湖和玄武湖 DOM 同步苂光光谱中 $235 、 275$ 和 $350 \mathrm{~nm}$ 处的峰苂光强度显著降低, 说明 TC 可以与 DOM 中的类色氨酸、类络氨酸和类腐殖质组分发生相互作用 (图 2a,b). 其中 $275 \mathrm{~nm}$ 处的 荧光猝灭较强, 表明类色氨酸荧光团对 DOM 的内在苂光猝灭贡献更大, 而 $350 \mathrm{~nm}$ 处的苂光强度变化最小, 说明类腐殖物质与 TC 的荧光猝灭作用有限. 此外, 同步荧光光谱也可一定程度上揭示 DOM 荧光基团微环 境的变化 ${ }^{[23]} .275 \mathrm{~nm}$ 处的荧光峰在 2 个 DOM 中随 TC 浓度添加都发生蓝移现象 $(275 \sim 270 \mathrm{~nm})$. 这可能是 由于类蛋白质中色氨酸残基附近的微环境极性降低引起 ${ }^{[23]}$. TC 存在时类色氨酸残基位于疏水性更强的微 环境中, 更少地暴露于溶液中. 文献表明, $\mathrm{TC}$ 分子主要黏附在色氨酸残基的疏水位点中 ${ }^{[24]}$. 可以看出, DOM 不同组分对 $\mathrm{TC}$ 的亲和力和黏附位点具有明显差异.

进一步研究 DOM 和 TC 的猝灭类型, 太湖和玄武湖 DOM 与 TC 相互作用后的 UV-Vis 光谱变化分别如图 2c 和 d 所示. $200 \mathrm{~nm}$ 附近的吸收峰表征多肽骨架结构, $210 ~ 310 \mathrm{~nm}$ 之间的吸收峰来源于色氨酸和酪氨酸中氨 基酸侧链的光吸收, 而 $356 \mathrm{~nm}$ 附近的吸收峰则是 $\mathrm{TC}$ 的分子特征 ${ }^{[25]}$. TC 添加前后 DOM 吸收光谱的剧烈变化 表明,TC 对 2 个 DOM 的苂光猝灭均属于静态猝灭 ${ }^{[10]}$. 静态猝灭是指苂光分子和猝灭剂作用形成非苂光络合 物的过程, 而猝灭剂通过碰撞和能量转移等使激发态猝灭分子返回基态的过程称为动态猝灭, 动态猝灭不会 改变吸收光谱. 以上结果表明 2 个湖泊 DOM 可以与 TC 发生络合作用,形成了非苂光性的 DOM-TC 络合物, 导 致自由游离态 $\mathrm{TC}$ 浓度降低. 文献指出,水体中抗生素的自由态浓度与其抗性基因表达呈正相关 ${ }^{[5]}$. 此外, DOM 也可能作为架桥促进 TC 在沉积物矿物界面的吸附, 促进 TC 从水相到固相界面的传输迁移 ${ }^{[4]}$.
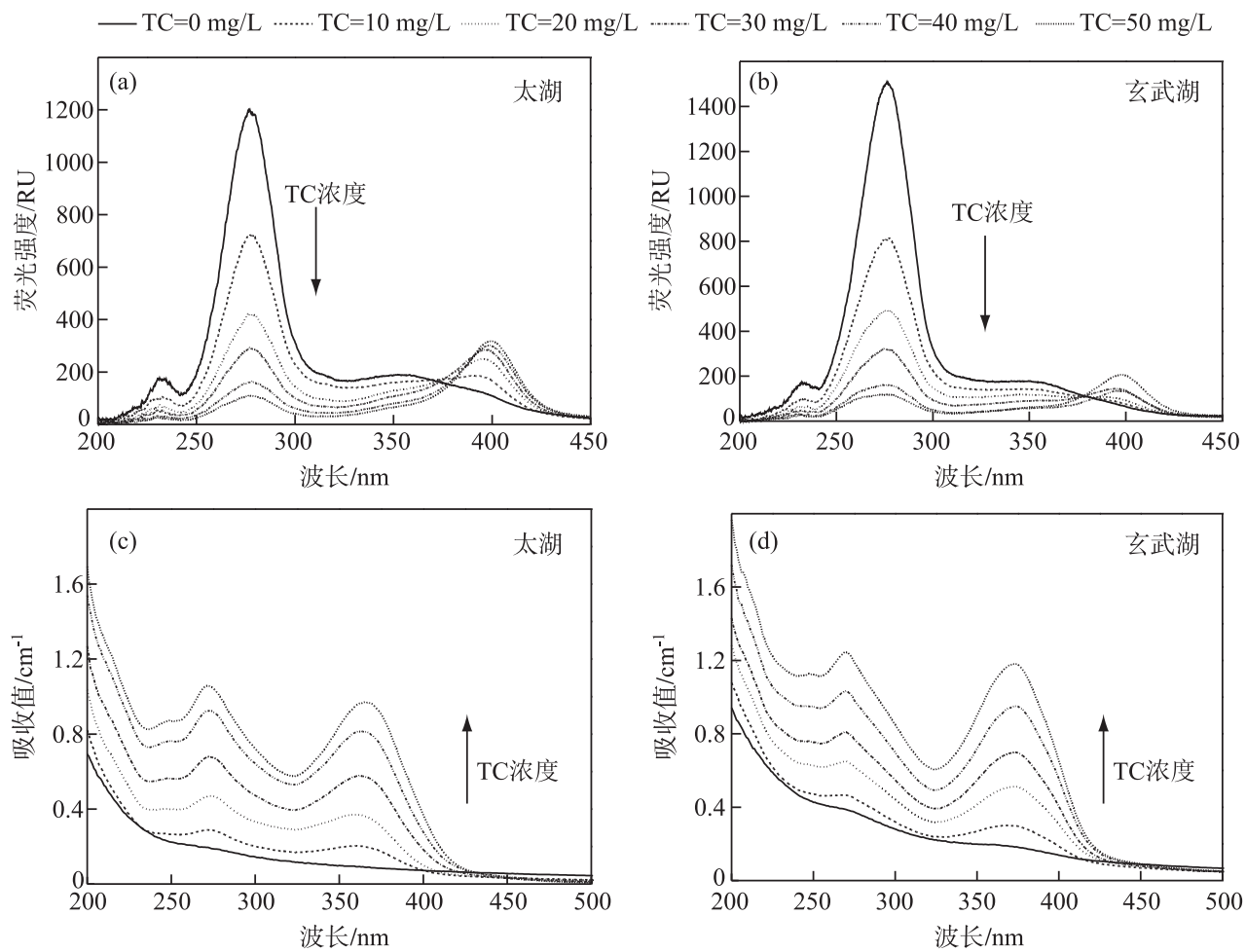

图 2 TC 对太湖和玄武湖 DOM 的同步苂光光谱 $(a, b)$ 和紫外光谱 $(c, d)$

Fig.2 Synchronous fluorescence spectra $(\mathrm{a}, \mathrm{b})$ and UV-Vis spectra(c,d) of TC-DOM interaction in Lake Taihu and Lake Xuanwu

\subsection{2 个湖泊 DOM 与 TC 荧光猝灭效应的二维相关光谱分析}

对 DOM 同步若光图谱进行 2D-COS 分析, 得到 TC 与不同 DOM 苂光组分的络合顺序.太湖 DOM 同步图 
的对角线上分别在 275 和 $400 \mathrm{~nm}$ 附近出现 2 个自交正峰(图 3a), 表明相应组分的光谱强度变化剧烈. 而位 于 $235 \mathrm{~nm} / 275 \mathrm{~nm}$ 和 $275 \mathrm{~nm} / 400 \mathrm{~nm}$ 附近的 2 个正交叉峰, 说明位于 $235 \mathrm{~nm}$ 的类络氨酸苂光峰、位于 275 $\mathrm{nm}$ 的类色氨酸苂光峰和位于 $400 \mathrm{~nm}$ 的类富里酸荧光峰光谱变化方向一致 (随 $\mathrm{TC}$ 浓度升高而降低). 根据 Noda 等规则 ${ }^{[18]}$, 异步图可揭示外部干扰下不同波长连续变化的程度: 位于波长 $\lambda 1 / \lambda 2$ 的正交叉峰表明 $\lambda 1$ 处的光谱变化比 $\lambda 2$ 处更快速; 位于波长 $\lambda 1 / \lambda 2$ 的负交叉峰表明 $\lambda 1$ 处的光谱变化落后于 $\lambda 2$ 处. 在 TDOM 异步图的对角线下方 (图 3b), $275 \mathrm{~nm} / 235 \mathrm{~nm}$ 和 $275 \mathrm{~nm} / 260 \mathrm{~nm}$ 处分别存在 2 个正交叉峰, 而 $400 \mathrm{~nm} / 235$ $\mathrm{nm} 、 400 \mathrm{~nm} / 275 \mathrm{~nm}$ 和 $400 \mathrm{~nm} / 350 \mathrm{~nm}$ 处分别存在 3 个负交叉峰, 这些图谱特征表明菼光猝灭顺序为: 275 $\mathrm{nm}>260 \mathrm{~nm}>235 \mathrm{~nm}>350 \mathrm{~nm}>400 \mathrm{~nm}$, 即苂光猝灭优先发生在低波长处. 因此, 与类腐殖物质相比, 类色 氨酸和类酪氨酸物质对 TC 的敏感性更高. 在玄武湖 DOM 中观察到了类似的现象 (图 $3 \mathrm{c}$ 和 d), 证明 TC 与 类蛋白物质的相互作用优先于类腐殖物质,与 DOM 来源无关.
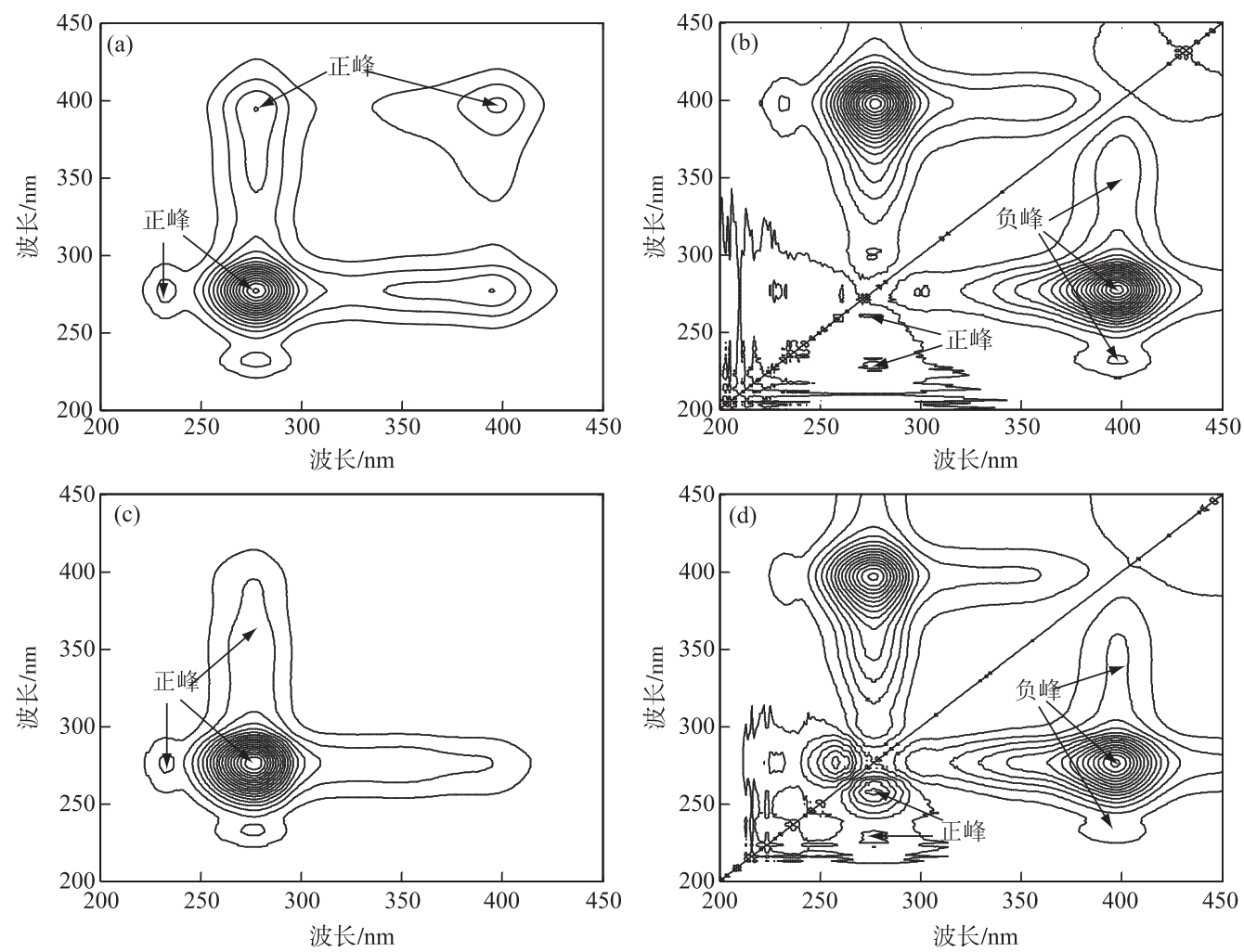

图 $3 \mathrm{DOM}$ 与 TC 相互作用的 2D-COS 图:

太湖 DOM 同步图 (a) 和异步图 (b) , 玄武湖 DOM 同步图 (c) 和异步图 (d)

Fig. 3 2D-COS plots of DOM-TC interaction: synchronous map(a) and asynchronous map (b) of DOM in Lake Taihu, synchronous $\operatorname{map}(\mathrm{c})$ and asynchronous $\operatorname{map}(\mathrm{d})$ of DOM in Lake Xuanwu

\subsection{2 个湖泊 DOM 荧光组分与 TC 的相互作用}

2.4.12 个湖泊 DOM 的 PARAFAC 组分特征 虽然同步荧光光谱可以定性表征 DOM 与 TC 的相互作用,但 不同苂光团的苂光峰可能重叠, 无法准确定量独立苂光组分与 TC 的相互作用特点. 通过 PARAFAC 分析模 型计算和 “S4T6C3” 检验验证, 共得到 3 种荧光组分. 如图 4 所示, 组分 C1 的发射最大值在 $425 \mathrm{~nm}$, 激发最 大值在 $250 \mathrm{~nm}$, 与类腐殖质中的富里酸苂光组分相似 ${ }^{[26]}$. 组分 $\mathrm{C} 2$ 存在 2 个苂光峰, 分别位于 $\mathrm{Ex} / \mathrm{Em} 230$ $\mathrm{nm} / 336 \mathrm{~nm}$ 和 $275 \mathrm{~nm} / 336 \mathrm{~nm}$, 与文献中经典的 $\mathrm{T}$ 峰一致 ${ }^{[27]}$, 代表类蛋白质中的色氨酸荧光组分. $C 3$ 的苂光 峰形状与 C2 类似, 有 2 个激发最大值分别位于 230 和 $260 \mathrm{~nm}, 1$ 个发射最大值位于 $303 \mathrm{~nm}$, 与文献中的 B 
峰位置相似 ${ }^{[27]}$, 为类蛋白质中的酪氨酸苂光组分. 根据 3 个组分的苂光得分 $\left(F_{\max }\right)$, 太湖 DOM 中 C1、C2 和 C3 分别占总荧光组分的 $18 \%$ 、66\% 和 16\%, 玄武湖 DOM 中相应组分分别占 $11 \% 、 65 \%$ 和 $24 \%$, 太湖 DOM 比 玄武湖 DOM 中类腐殖组分含量更高.
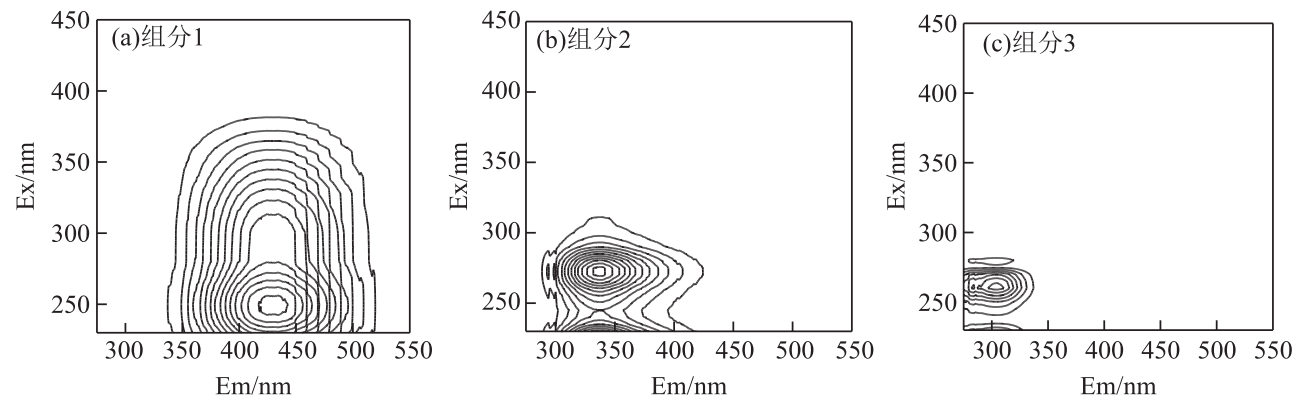

图 4 PARAFAC 苂光组分的苂光光谱图

Fig.4 Excitation-emission matrix spectra of PARAFAC fluorescent components

2.4.2 2 个湖泊 DOM 荧光组分猝灭 3 个 PARAFAC 组分对 TC 表现出不同的响应特征 (图 5). 在太湖 DOM 中, 添加少量的 $\mathrm{TC}(6.5 \mu \mathrm{mol} / \mathrm{L})$ 显著降低 $\mathrm{C} 2$ 和 $\mathrm{C} 3$ 的苂光强度, 而 $\mathrm{C} 1$ 的苂光猝灭程度相对较弱. 随着 TC 浓度增加, 3 种组分的 $F_{\text {max }}$ 逐渐降低, 当 $\mathrm{TC}$ 浓度为 $45.5 \mu \mathrm{mol} / \mathrm{L}$ 时, C1、C2 和 C3 组分的可猝灭部分分别为 $79 \% 、 100 \%$ 和 $99 \%$. 玄武湖 DOM 的结果相似, C1、C2 和 C3 组分的可猝灭部分分别为 $82 \%$ 、98\% 和 $96 \%$,表明 $\mathrm{C} 1$ 与 $\mathrm{TC}$ 的相互作用弱于 $\mathrm{C} 2$ 和 C3. 因此, TC 络合特征与 DOM 独立组分密切相关. 文献指出在同样温度范 围标准色氨酸的猝灭程度高于标准腐殖质的猝灭效应 ${ }^{[28]}$, 但另一项研究也发现 L-络氨酸 ( $52 \%$ ) 比 L-色氨 酸 $(35 \%)$ 的苂光猝灭更强 ${ }^{[29]}$.
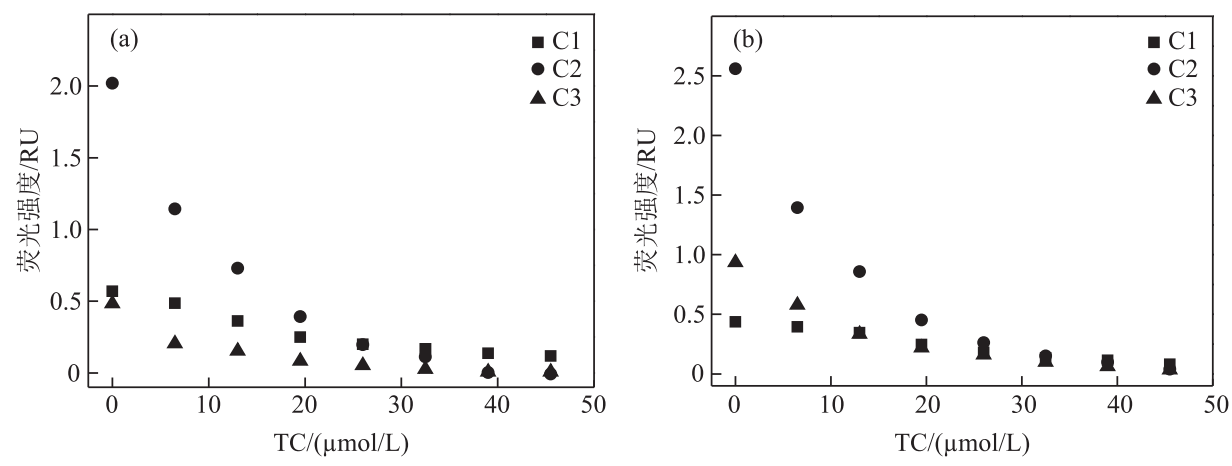

图 5 太湖 (a) 和玄武湖 (b) DOM 中 TC 与 3 个 PARAFAC 苂光组分的苂光猝灭图

Fig.5 Fluorescence quenching diagram of TC with three PARAFAC fluorescence components in Lake Taihu(a) and Lake Xuanwu(b)

通过 Ryan-Weber 非线性模型拟合 3 个荧光组分的猝灭过程获得络合常数,如表 1 所示, $R^{2}$ 表明该模型 拟合度很高. 在太湖和玄武湖 DOM 中 3 种荧光组分的 $\lg K$ 值范围分别为 $5.29 \sim 5.73$ 和 $5.05 \sim 5.85$, 表明 TC 与 DOM 发生了强烈的络合作用. 2 个湖泊 DOM 中 C2、C3 的 $F_{\text {end }}$ 值均为 0 , 表明类蛋白质组分几乎完全被络 合,而太湖和玄武湖 DOM 中分别有 $9.78 \%$ 和 $16.78 \%$ 的 C1 组分未与 TC 发生络合作用. 相应的,3 个苂光组 分的 $\lg K$ 值在 2 个 DOM 中大小顺序相同, 均表现为 $\mathrm{C} 2>\mathrm{C} 3>\mathrm{C} 1$, 表明类蛋白质更易与 TC 形成络合物. 在 DOM 与磺胺二甲基嘧啶的相互作用中发现了类似的结果 ${ }^{[4]}$, 进一步采用标准色氨酸、标准络氨酸和标准腐 殖质作为典型 DOM, 发现抗生素对色氨酸和络氨酸的苂光猝灭相似, 均强于腐殖质 ${ }^{[30]}$. 这可能是因为腐殖 
质主要以酚基、芳基和羧基为主,蛋白质主要以氨基和脂肪族为主, 而氨基 I、氨基 II、芳香族和脂肪族是 DOM 与 TC 反应过程中的作用官能团 ${ }^{[13]}$. 然而, 也有研究发现 DOM 与卡马西平的相互作用中类富里酸物质 的络合作用更强 ${ }^{[3]}$, 说明不同的 DOM 种类和猝灭剂的络合作用存在差异. 此外, 虽然太湖 DOM 中 C1 比例 高于 C3, 但 $\mathrm{C} 1$ 的 $\lg K$ 值小于 $\mathrm{C} 3$, 说明组分络合作用与其在 DOM 中的比例无关. C2 的 $\lg K$ 值大于 C3 , 说明 类色氨酸比类络氨酸物与 TC 作用更强, 这与 $2 \mathrm{D}-\mathrm{COS}$ 结果一致, 即 $275 \mathrm{~nm}$ 处的苂光猝灭优先于 $235 \mathrm{~nm}$ 处. 总体来说, 富含类色氨酸和类络氨酸组分的 DOM 能有效地与 TCs 发生络合作用, 形成复合物, 降低 TCs 的 生物有效性. 然而, DOM 具有生物活性和光活性, 在自然矿化过程中容易被生物降解和光解, 可能导致络合 态 TCs 重新进人水体中,影响 TCs 在水环境中的迁移以及对植物和生物的有效性和毒性.

表 1 Ryan-Weber 模型计算的太湖和玄武湖 DOM 中 3 个 PARAFAC 组分与 TC 相互作用的 $\lg K$ 和 $F_{\text {end }}$

Tab. 1 The $\lg K$ and $F_{\text {end }}$ for the three PARAFAC components in DOM of

Lake Taihu and Lake Xuanwu calculated by Ryan-Weber model

\begin{tabular}{|c|c|c|c|c|c|c|c|c|}
\hline \multirow{2}{*}{$\begin{array}{l}\text { PARAFAC } \\
\text { 组分 }\end{array}$} & \multicolumn{4}{|c|}{ 太湖 DOM } & \multicolumn{4}{|c|}{ 玄武湖 DOM } \\
\hline & $\lg K$ & $\begin{array}{c}C_{\mathrm{L}} / \\
(\mu \mathrm{mol} / \mathrm{L})\end{array}$ & $F_{\text {end }}$ & $R^{2}$ & $\lg K$ & $\begin{array}{c}C_{\mathrm{L}} / \\
(\mu \mathrm{mol} / \mathrm{L})\end{array}$ & $F_{\text {end }}$ & $R^{2}$ \\
\hline C1 & 5.29 & 43.02 & 9.78 & 0.9788 & 5.05 & 28.76 & 16.78 & 0.9922 \\
\hline $\mathrm{C} 2$ & 5.73 & 13.39 & 0 & 0.9926 & 5.85 & 16.13 & 0 & 0.9859 \\
\hline $\mathrm{C} 3$ & 5.58 & 14.34 & 0 & 0.9963 & 5.57 & 6.90 & 0 & 0.9785 \\
\hline
\end{tabular}

\section{3 结论}

1) EEM-PARAFAC 分析显示太湖和玄武湖 DOM 中均含有 3 个苂光组分, 其中类色氨酸组分 C2 是 2 个 DOM 的主要苂光组成, 其次为类络氨酸 $\mathrm{C} 3$ 和类富里酸 $\mathrm{C} 1$ 组分.

2) 同步苂光光谱联合 2D-COS 分析表明类色氨酸优先于类络氨酸物质与 TC 发生猝灭作用, 而类富里酸 组分对 TC 敏感性最低.

3) DOM 与 TC 的猝灭机制属于静态猝灭,TC 可改变 DOM 分子的微环境极性. 3 个苂光组分与 TC 的苂 光猝灭符合 Ryan-Weber 非线性方程, 并且各组分与 TC 的络合常数大小顺序为: C2 > C3 > C1.

\section{4 参考文献}

[ 1 ] Chen XF, Chuai XM, Liu T et al. Characteristics and source identification of the dissolved organic matter in the lakes of west Jiangsu by spectroscopy. J Lake Sci, 2012, 24(2) : 259-266. DOI: 10.18307/2012.0214. [陈小锋, 揣小明, 刘涛 等. 江苏省西部湖泊溶解性有机物光谱学特征和来源解析. 湖泊科学, 2012, 24(2): 259-266.]

[ 2 ] Yao X, Zhang YL, Zhu GW et al. Different degradation mechanism of dissolved organic matter derived from phytoplankton and macrophytes in Lake Taihu, China. Acta Scientiae Circumstantiae, 2014, 34(3): 688-694. [姚昕, 张运林, 朱广伟 等. 湖泊草、藻来源溶解性有机质及其微生物降解的差异. 环境科学学报, 2014, 34(3) : 688-694.]

[ 3 ] Hernandez-Ruiz S, Abrell L, Wickramasekara S et al. Quantifying PPCP interaction with dissolved organic matter in aqueous solution: combined use of fluorescence quenching and tandem mass spectrometry. Water Research, 2012, 46(4) : 943-954.

[ 4 ] Bai LL, Cao CC, Wang CH et al. Roles of phytoplankton-and macrophyte-derived dissolved organic matter in sulfamethazine adsorption on goethite. Environmental Pollution, 2017, 230: 87-95.

[ 5 ] Chen ZY, Zhang YJ, Gao YZ et al. Influence of dissolved organic matter on tetracycline bioavailability to an antibiotic-resistant bacterium. Environmental Science \& Technology, 2015, 49(18) : 10903-10910.

[6] Sarmah AK, Meyer MT, Boxall ABA. A global perspective on the use, sales, exposure pathways, occurrence, fate and effects of veterinary antibiotics (VAs) in the environment. Chemosphere, 2006, 65(5) : 725-759.

[ 7 ] Bai LL, Xu HC, Wang CH et al. Extracellular polymeric substances facilitate the biosorption of phenanthrene on cyanobacteria Microcystis aeruginosa. Chemosphere, 2016, 162: 172-180.

[ 8 ] Zhu JP, Mei T, Peng Y et al. Characterizing the interaction between roxarsone and humic acid by fluorescence quenching experiment. Environmental Science, 2014, 35(7) : 2620-2626. [ 朱江鹏, 梅婷, 彭云等. 苂光猝灭法研究洛克沙胂与 
腐殖酸的相互作用. 环境科学, 2014, 35(7) : 2620-2626. ]

[ 9 ] Huang CC, Li YM, Wang Q et al. Components optical property of CDOM in Lake Taihu based on three-dimensional excitation emission matrix fluorescence. J Lake Sci, 2010, 22(3) : 375-382. DOI: 10.18307/2010.0309. [黄昌春, 李云梅, 王桥 等. 基于三维荧光和平行因子分析法的太湖水体 CDOM 组分光学特征. 湖泊科学, 2010, 22(3) : 375-382.]

[10] Xu J, Sheng GP, Ma Y et al. Roles of extracellular polymeric substances (EPS) in the migration and removal of sulfamethazine in activated sludge system. Water Research, 2013, 47(14) : 5298-5306.

[11] Xu HC, Yu GH, Yang LY et al. Combination of two-dimensional correlation spectroscopy and parallel factor analysis to characterize the binding of heavy metals with DOM in lake sediments. Journal of Hazardous Materials, 2013, 263: 412-421.

[12] Chen W, Habibul N, Liu XY et al. FTIR and synchronous fluorescence heterospectral two-dimensional correlation analyses on the binding characteristics of copper onto dissolved organic matter. Environmental Science \& Technology, 2015, 49(4) : 2052-2058.

[13] Bai LL, Zhao Z, Wang CL et al. Multi-spectroscopic investigation on the complexation of tetracycline with dissolved organic matter derived from algae and macrophyte. Chemosphere, 2017, 187: 421-429.

[14] Yan PF, Hu ZH, Yu HQ et al. Fluorescence quenching effects of antibiotics on the main components of dissolved organic matter. Environmental Science and Pollution Research, 2016, 23(6) : 5667-5675.

[15] Xu J, Zhang Y, Zhou CB et al. Distribution, sources and composition of antibiotics in sediment, overlying water and pore water from Taihu Lake, China. Science of the Total Environment, 2014, 497/498: 267-273.

[16] Zhang SH, Pang S, Wang PF et al. Antibiotic concentration and antibiotic-resistant bacteria in two shallow urban lakes after stormwater event. Environmental Science and Pollution Research, 2016, 23(10) : 9984-9992.

[17] Murphy KR, Stedmon CA, Graeber D et al. Fluorescence spectroscopy and multi-way techniques. PARAFAC. Analytical Methods, 2013, 5(23) : 6557-6566.

[18] Noda I, Ozaki Y. Two-dimensional correlation spectroscopy : applications in vibrational and optical spectroscopy. London: John Wiley and Sons Inc, 2005.

[19] Ryan DK, Weber JH. Fluorescence quenching titration for determination of complexing capacities and stability constants of fulvic acid.Analytical Chemistry, 1982, 54(6) : 986-990.

[20] Ishii SKL, Boyer TH. Behavior of reoccurringPARAFAC components in fluorescent dissolved organic matter in natural and engineered systems: A critical review. Environmental Science \& Technology, 2012, 46(4) : 2006-2017.

[21] Fu PQ, Wu FC, Liu CQ et al. Three-dimensional excitation emission matrix fluorescence spectroscopy of dissolved organic matter from Chinese highland lakes. Oceanologia et Limnologia Sinica, 2007, 38(6) : 512-520. [傅平青, 吴丰昌, 刘丛 强等. 高原湖泊溶解有机质的三维苂光光谱特性初步研究. 海洋与湖沼, 2007, 38(6) : 512-520.]

[22] Li D, Li XW, Niu ZC et al. A comparative study on macrobenthic community structure and diversity in different trophic status lakes of Jiangsu Province. Asian Journal of Ecotoxicology, 2017, 12(1): 163-172. [李娣, 李旭文, 牛志春等. 江 苏省不同营养状况湖泊底栖动物群落结构与多样性比较. 生态毒理学报, 2017, 12(1): 163-172.]

[23] Bani-Yaseen AD. Spectrofluorimetric study onthe interaction between antimicrobial drug sulfamethazine and bovine serum albumin. Journal of Luminescence, 2011, 131(5) : 1042-1047.

[24] Anand U, Jash C, Boddepalli RK et al. Exploring the mechanism of fluorescence quenching in proteins induced by tetracycline. The Journal of Physical Chemistry B, 2011, 115(19) : 6312-6320.

[25] Bi HN, Tang L, Gao X et al. Spectroscopic analysis on the binding interaction between tetracycline hydrochloride and bovine proteins $\beta$-casein, $\alpha$-lactalbumin. Journal of Luminescence, 2016, 178: 72-83.

[26] Lee BM, Seo YS, Hur J. Investigation of adsorptive fractionation of humic acid on graphene oxide using fluorescence EEMPARAFAC. Water Research, 2015, 73: 242-251.

[27] Yamashita Y, Jaffé R, Maie N et al. Assessing the dynamics of dissolved organic matter (DOM) in coastal environments by excitation emission matrix fluorescence and parallel factor analysis (EEM-PARAFAC). Limnology and Oceanography, $2008, \mathbf{5 3}(5)$ : 1900-1908

[28] Baker A. Thermal fluorescence quenchingproperties of dissolved organic matter. Water Research, 2005, 39(18) : 4405-4412.

[29] Wang ZG, Cao J, Meng FG. Interactions between protein-like and humic-like components in dissolved organic matter revealed by fluorescence quenching. Water Research, 2015, 68: 404-413.

[30] Peng N, Wang KF, Liu GG et al. Quantifying interactions between propranolol and dissolved organic matter (DOM) from different sources using fluorescence spectroscopy. Environmental Science and Pollution Research, 2014, 21 ( 7 ): $5217-5226$. 